\title{
Avaliação do consumo alimentar de pacientes com mucopolissacaridose
}

\author{
Assessment of dietary intake of patients with mucopolysaccharidosis
}

\author{
Roberta B. Leite1, Maria Efigênia Q. Leite², Júlia D. D. Freitas³, Carlos M. C. Mendes ${ }^{4}$, Tereza C. B. J. Deirón \\ Angelina X. Acosta ${ }^{6}$
}

${ }^{1}$ Especialista em Nutrição Hospitalar com ênfase em Saúde da Criança, Programa de Residência Multiprofissional em Saúde, Complexo Hospitalar Universitário Professor Edgard Santos (COM-HUPES), Universidade Federal da Bahia (UFBA), Salvador, BA; ${ }^{2}$ Doutoranda em Medicina e Saúde pela Faculdade de Medicina da UFBA, Mestre em Alimentos, Nutrição e Saúde, Escola de Nutrição, UFBA, Salvador, BA; ${ }^{3}$ Especialista em Nutrição Clínica, Programa de Pós Graduação sob a forma de Residência da Escola de Nutrição, UFBA, Salvador, BA; ${ }^{4}$ Doutor em Saúde Coletiva com área de concentração em Epidemiologia, Instituto de Ciências da Saúde, Programa de Pós-graduação em Processos Interativos de Órgãos e Sistemas, UFBA, Salvador, BA; ${ }^{5}$ Doutora em Nutrição, Universidade Federal de Pernambuco (UFPE), Recife, PE; ${ }^{6}$ Doutora em Clínica Médica, Faculdade de Medicina de Ribeirão Preto (FMRP), Universidade de São Paulo (USP), Ribeirão Preto, SP.

\section{RESUMO}

Objetivos: Avaliar o consumo alimentar de crianças e adolescentes com mucopolissacaridose.

Métodos: Série de casos de mucopolissacaridose acompanhados regularmente em um serviço de referência da cidade de Salvador, Bahia, no período de janeiro a abril de 2012. Foram considerados para inclusão pacientes de ambos os sexos, entre dois e 18 anos de idade, com ingestão alimentar por via oral, sem complicações clínicas. Foi aplicada anamnese estruturada contemplando informações socioeconômicas, clínicas e avaliação da ingestão alimentar. Dados de consumo alimentar foram obtidos através do recordatório alimentar de 24 horas e registro alimentar de três dias. Foram avaliadas as possíveis inadequações alimentares através das recomendações da Dietary Reference Intakes, 2005. Os dados foram tabulados no Epidata 3.1 e analisados no pacote estatístico R.

Resultados: Foram estudados oito meninos e duas meninas, com idade mediana de 10 anos (intervalo interquartil 6,4 anos, mínima 3, máxima 16 anos). O tipo mais frequente da doença foi a mucopolissacaridose VI (60\%). Seis pacientes necessitavam de auxílio para se alimentar, nove apresentaram baixa ingestão de calorias e seis apresentaram baixa ingestão de lipídios. Todos os pacientes apresentavam insuficiente ingestão de fibras e consumo adequado de carboidratos e proteínas. O consumo de todos os micronutrientes apresentou inadequação.

Conclusões: Detectou-se alta frequência de inadequação no consumo alimentar de crianças e adolescentes com mucopolissacaridose. Algumas limitações osteoarticulares causadas pela doença acarretam problemas na ingestão de alimentos, tendo muitos pacientes necessidade de auxílio no ato de se alimentar. A conduta nutricional especializada poderá auxiliar na qualidade de vida e no prognóstico desses indivíduos.

DESCRITORES: MUCOPOLISSACARIDOSE; CONSUMO ALIMENTAR; INADEQUAÇÕES ALIMENTARES.

\section{ABSTRACT}

Aims: To assess the dietary intake of children and adolescents with mucopolysaccharidosis.

Methods: Series of cases of mucopolysaccharidosis regularly followed in a referral center in Salvador, Bahia state, Brazil, in January-April 2012. Patients of both sexes aged between 2 and 18 years with food intake by mouth and without clinical complications were considered to inclusion. Structured anamnesis was applied contemplating socioeconomic, clinical, and dietary intake information. The food consumption data were obtained from 24-hour dietary recall and food record of three days. Possible dietary inadequacies were evaluated facing the recommendations of the Dietary Reference Intakes, 2005. Data were tabulated in 3.1 Epidata and analyzed by the statistical package R.

Results: Eight boys and two girls, with a median age of 10 years (interquartile range 6.4 years, minimum 3, maximum 16 years) were studied. The most common type of the disease was Mucopolysaccharidosis VI (60\%) and less frequent mucopolysaccharidosis I (10\%). Six patients needed assistance to feed, nine had low calorie intake, and six had low intake of lipids. All patients had insufficient intake of fiber and adequate intake of carbohydrates and proteins. The intake of all micronutrients had impairments.

Conclusions: There was a high frequency of inadequate dietary intake in children and adolescents with mucopolysaccharidosis. Some osteoarticular limitations caused by the disease lead to problems in food intake, and many patients need assistance in the act of eating. A specialized nutritional intervention may assist in quality of life and prognosis of these individuals.

KEY WORDS: MUCOPOLYSACCHARIDOSIS; FOOD CONSUMPTION; DIETARY INADEQUACIES 


\section{INTRODUÇÃO}

As mucopolissacaridoses (MPS) compreendem um conjunto de doenças genéticas de depósito lisossomal, causadas pela deficiência de uma das onze enzimas lisossomais responsáveis pela degradação dos glicosaminoglicanos (GAG). ${ }^{1,2}$ Fragmentos de GAG parcialmente degradados se acumulam nos lisossomos, resultando em anormalidades clínicas, determinando um quadro clínico multissistêmico, crônico e progressivo. ${ }^{3}$

As MPS são diferenciadas bioquimicamente pela deficiência da atividade da enzima associada, sendo classificadas como tipo I (com três subtipos denominados Síndrome de Hurler, Hurler-Scheie e Scheie), tipo II (Síndrome de Hunter), tipo III (com quatro subtipos denominados Síndrome de Sanfilippo A, B, C, D), tipo IV (com dois subtipos denominados Síndrome de Morquio A, B), Tipo VI (MaroteauxLamy), tipo VII (Sly) e tipo IX (Notowicks). ${ }^{4}$

A incidência estimada total, incluindo todos os tipos MPS é de aproximadamente $01 \mathrm{em} 20.000$ nascimentos e de 01 em 100.000 nascimentos isoladamente. ${ }^{4}$ No Brasil o tipo de MPS VI é mais frequentemente diagnosticado, com maior frequência no Sudeste seguida da região Nordeste. ${ }^{1}$ Em um estudo realizado na cidade de Monte Santo (Bahia/Brasil) a MPS tipo VI apresentou um número de casos muito maior do que descrito pela literatura, com mais de 01 em cada 5.000 habitantes, possivelmente devido à endogamia elevada nesta região. ${ }^{5}$

Ao nascimento, a maioria das crianças não possui qualquer indício de anormalidades anatômicas ou fisiológicas, no entanto deformidades progressivas surgem à medida que ocorre o acúmulo de GAG. ${ }^{6}$

A rigidez das articulações é comum em todas as formas de MPS, e o espectro máximo de movimentos pode ser limitado, principalmente em ombros e braços. As mãos são curtas e largas, com dedos que gradualmente vão ficando encurvados e rígidos, tornando difícil a realização de atividades habituais e simples como o ato de se alimentar. ${ }^{7} \mathrm{Na}$ cavidade oral podem ser visualizadas alterações como a macroglossia, dentição displásica, alterações de oclusão e hiperplasia gengival, capazes de interferir na ingestão adequada dos alimentos, limitar a autonomia do ato de se alimentar e consequentemente impactar negativamente no estado nutricional desses pacientes. ${ }^{8}$

A capital baiana possui um Hospital Universitário (Complexo Hospitalar Universitário Professor Edgard Santos/COM-HUPES), que atende em seu Serviço de Genética os pacientes com MPS da região, destacando-se pelo acompanhamento e tratamento multiprofissional desses pacientes. A nutrição está inserida nesse contexto, no intuito de conhecer, prestar assistência e minimizar os efeitos da doença sobre o estado nutricional desses indivíduos. A escassez de estudos científicos nessa área do conhecimento da saúde tornam necessárias a elaboração e realização de trabalhos de pesquisa que busquem avaliar e descrever os aspectos alimentares e nutricionais envolvidos na evolução da doença, para o aprimoramento da conduta e manejo dietético especializado e individualizado. ${ }^{9}$

Dessa forma, o objetivo principal deste estudo, foi o de avaliar o consumo alimentar de pacientes com MPS acompanhados em um Serviço de Referência do estado da Bahia, na cidade de Salvador.

\section{MÉTODOS}

Esta série de casos apresenta 10 pacientes acompanhados regularmente em um serviço de referência da cidade de Salvador, Bahia, no período de janeiro a abril de 2012. Foram avaliados concomitantemente: 1) pacientes internados na enfermaria de pediatria do Complexo Hospitalar Universitário Professor Edgard Santos (COM-HUPES; 2) pacientes atendidos no Ambulatório de Genética do Magalhães Neto da Universidade Federal da Bahia; 3) Pacientes atendidos no domicílio em atividade de campo, todos acompanhados pelo Serviço de genética médica do referido hospital. O trabalho foi aprovado pelo Comitê de Ética em Pesquisa do COM-HUPES (Protocolo CEP/HUPES $n^{\circ} 124.2011$ ).

Foram considerados elegíveis para o estudo crianças e adolescentes de ambos os sexos, com idade entre dois e 18 anos, com diagnóstico clínico e laboratorial de MPS, com ingestão alimentar por via oral, sem complicações clínicas (traqueostomia, gastrostomia, insuficiência renal aguda ou crônica, cardiopatia grave, desnutrição energético-proteica grave) e que tiveram sua participação no estudo consentida pelos pais e/ ou responsáveis através da assinatura do Termo de Consentimento Livre e Esclarecido. Um total de 19 pacientes foi avaliado, dos quais seis não entregaram o registro alimentar de três dias, um recusou-se a participar e dois não preenchiam os critérios de inclusão do estudo (um paciente tinha idade superior a 18 anos e o outro estava com desnutrição energéticoproteica grave) sendo a amostra final composta por 10 pacientes.

Foi aplicada uma anamnese que contemplou dados socioeconômicos (renda e escolaridade), dados clínicos obtidos do prontuário médico, avaliação da ingestão 
alimentar (consistência e quantidade de alimentos, autonomia em se alimentar), sendo investigada a perda ponderal não intencional nos últimos seis meses. ${ }^{9}$ Para a aferição do peso do paciente foi utilizada uma balança digital da marca Wiso, modelo W801, com capacidade máxima para $180 \mathrm{~kg} .{ }^{10}$

As informações sobre o consumo alimentar foram coletadas utilizando os instrumentos: 1) recordatório alimentar de 24 horas, aplicado pelo pesquisador e 2) registro alimentar de três dias (referente a dois dias da semana e um dia do final de semana). ${ }^{11}$ Um álbum fotográfico foi utilizado no auxílio do preenchimento das informações, no intuito de minimizar o viés de memória. ${ }^{12} \mathrm{O}$ preenchimento do registro alimentar de três dias ocorreu no domicílio do participante conforme orientação prévia, sendo este posteriormente devolvido no retorno dos pacientes ao ambulatório. Os pacientes sem alterações cognitivas forneceram diretamente as informações e as demais foram preenchidas pelos responsáveis.

As quantidades consumidas dos alimentos, reportadas em medidas caseiras, foram transformadas em gramas ou mililitros, e posteriormente avaliadas por meio do Software de Nutrição AVANUTRI versão $3.11,{ }^{13}$ obtendo-se o cálculo da composição centesimal de calorias, macronutrientes (carboidratos, proteína e lipídios) e micronutrientes (vitaminas e minerais). Para a avaliação da adequação de calorias, macronutrientes e micronutrientes, foram reunidos os valores médios de ingestão, sendo a adequação definida através das recomendações estabelecidas pela Dietary Reference Intakes (DRIs), 2005. ${ }^{14}$ Foram utilizadas as referências nutricionais para a população saudável devido à ausência na literatura de referências específicas para indivíduos com MPS.

Todos os dados foram tabulados no Epidata, versão $3.1 .^{15}$ Por tratar-se de um plano amostral não probabilístico e, consequentemente, não sendo possível uma estimativa adequada do erro-padrão, não foram calculadas estatísticas inferenciais. ${ }^{16}$ Foram obtidas as estatísticas descritivas para as variáveis do estudo conforme o nível de mensuração das mesmas e a falta de aderência à distribuição normal teórica (mediana, intervalo interquartílico e proporções). As análises foram realizadas no pacote estatístico R. ${ }^{17}$

\section{RESULTADOS}

Dos 10 pacientes com MPS avaliados, quatro estavam internados na enfermaria de pediatria do COM-HUPES, dois pacientes foram atendidos no ambulatório de Genética Médica do COM-HUPES e quatro foram atendidos em seus domicílios durante atividade de campo. Em relação à idade dos pacientes, a variável mostrou-se heterogênea (Coeficiente de Variação $=62,2 \%$ ), sendo a idade mínima de três anos e máxima de 16 anos, com idade mediana de 10 anos (intervalo interquartil $=6,4$ anos).

Observou-se maior frequência da MPS do tipo VI $(6 / 10,60 \%)$ e menor frequência da MPS do tipo I $(1 / 10,10 \%)$. Houve predomínio de indivíduos do sexo masculino $(8 / 10,80 \%)$. Em relação à renda familiar, $40 \%$ dos indivíduos entrevistados viviam com recurso de menos de dois salários mínimos. Os dados de escolaridade materna e paterna também estão descritos na Tabela 1.

Tabela 1. Caracterização dos pacientes com mucopolissacaridose de um serviço de referência de Salvador, estado da Bahia, Brasil $(\mathrm{N}=10)$.

\begin{tabular}{|c|c|c|}
\hline & N & $\%$ \\
\hline \multicolumn{3}{|l|}{ Diagnóstico Clínico } \\
\hline Mucopolissacaridose Tipo I - Síndrome de Hurler & 01 & 10 \\
\hline Mucopolissacaridose Tipo II - Síndrome de Hunter & 03 & 30 \\
\hline $\begin{array}{l}\text { Mucopolissacaridose Tipo VI - Síndrome de } \\
\text { Maroteaux-Lamy }\end{array}$ & 06 & 60 \\
\hline \multicolumn{3}{|l|}{ Sexo } \\
\hline Masculino & 08 & 80 \\
\hline Feminino & 02 & 20 \\
\hline \multicolumn{3}{|l|}{ Renda } \\
\hline$\leq 02$ salários mínimos* & 04 & 40 \\
\hline >02 salários mínimos & 06 & 60 \\
\hline \multicolumn{3}{|l|}{ Escolaridade Materna } \\
\hline Ensino fundamental & 06 & 60 \\
\hline Ensino médio & 02 & 20 \\
\hline Ensino superior & 02 & 20 \\
\hline \multicolumn{3}{|l|}{ Escolaridade Paterna } \\
\hline Analfabeto & 02 & 20 \\
\hline Ensino fundamental & 03 & 30 \\
\hline Ensino médio & 03 & 30 \\
\hline Ensino superior & 02 & 20 \\
\hline \multicolumn{3}{|l|}{ Ingestão alimentar } \\
\hline Tem dificuldade para usar utensílios & 01 & 10 \\
\hline Tem dificuldade para levar a comida à boca & 01 & 10 \\
\hline Tem dificuldade para levar o copo à boca & 01 & 10 \\
\hline Precisa de auxílio para se alimentar & 06 & 60 \\
\hline Precisa de adaptações nos utensílios para se alimentar & 01 & 10 \\
\hline Alteração na quantidade ou consistência dos alimentos & 02 & 20 \\
\hline
\end{tabular}

* Salário mínimo brasileiro (R\$ 622,00 na época da pesquisa).

Este estudo identificou que seis dos 10 pacientes $(60 \%)$ apresentavam dificuldade para usar utensílios (talheres, copos) e precisavam de auxílio para se 
alimentar, sugerindo possíveis prejuízos na ingestão alimentar decorrentes do comprometimento motor e funcional causados pela doença. Verificou-se que um paciente apresentava redução na quantidade de alimentos, e outro, alteração na consistência dos alimentos ingeridos, por conta das limitações impostas pela doença (Tabela 1).

Analisando-se a inadequação do consumo alimentar, verificou-se elevada frequência de indivíduos com baixo consumo de calorias, lipídios e fibras $(90 \%$, $60 \%$ e $100 \%$, respectivamente), entretanto não foram observadas inadequações na ingestão de proteínas e carboidratos (Tabela 2).

Tabela 2. Avaliação da inadequação do consumo alimentar (energia, macronutrientes e fibras) de acordo com as recomendações das Dietary Reference Intakes 2005, dos pacientes com mucopolissacaridose de um serviço de referência de Salvador, estado da Bahia, Brasil $(\mathrm{N}=10)$.

\begin{tabular}{lcc} 
& $\mathbf{n} * \mathbf{N} * *$ & $\%$ \\
Calorias & $\mathbf{0 9} / \mathbf{1 0}$ & $\mathbf{9 0}$ \\
Faixa etária & & \\
$(\geq 03-<09)$ & $02 / 03$ & 66,7 \\
$(\geq 09-<14)$ & $04 / 04$ & 100 \\
$(\geq 14-\leq 18)$ & $03 / 03$ & 100 \\
\hline Macronutrientes & & \\
Proteina & $00 / 10$ & 00 \\
Carboidrato & $00 / 10$ & 00 \\
Lipídios & $\mathbf{0 6} / \mathbf{1 0}$ & $\mathbf{6 0}$ \\
Faixa etária & & \\
$(\geq 01-<04)$ & $02 / 02$ & 100 \\
$(\geq 04-\leq 18)$ & $04 / 08$ & 50 \\
Fibra & $\mathbf{1 0} / \mathbf{1 0}$ & $\mathbf{1 0 0}$ \\
Faixa etária & & \\
$(\geq 01-<04)$ & $02 / 02$ & 100 \\
$(\geq 04-<09)$ & $01 / 01$ & 100 \\
$(\geq 09-<14)$ & $04 / 04$ & 100 \\
$(\geq 14-\leq 18)$ & $03 / 03$ & 100 \\
\hline
\end{tabular}

* Número de indivíduos com inadequação para cada categoria.

** Número total de indivíduos por categoria.

$\mathrm{Na}$ análise da ingestão de minerais, todos os pacientes apresentaram inadequação na ingestão diária de potássio, o baixo consumo de cálcio foi observado em $60 \%$ dos indivíduos, sendo o selênio, o mineral com menor frequência de inadequação ( $20 \%$ dos pacientes). Houve um consumo inadequado de grande parte das vitaminas nas diferentes faixas etárias, sendo que para as vitaminas do complexo B somente um paciente apresentou consumo inadequado (Tabela 3).
Tabela 3. Avaliação da inadequação do consumo alimentar dos micronutrientes (minerais e vitaminas), de acordo com as DRIs 2005, dos pacientes com MPS de um serviço de referência de Salvador-BA/Brasil $(N=10)$.

\begin{tabular}{|c|c|c|c|c|c|}
\hline Minerais & $\mathbf{n} * / \mathbf{N}^{* *}$ & $\%$ & Vitaminas & $\mathbf{n} * / \mathbf{N}^{* *}$ & $\%$ \\
\hline Cálcio (mg) & $06 / 10$ & 60 & Vitamina A (RE) & $10 / 04$ & 40 \\
\hline Faixa etária & & & Faixa etária & & \\
\hline$(\geq 01-<04)$ & $00 / 02$ & 00 & $(\geq 01-<04)$ & $02 / 00$ & 00 \\
\hline$(\geq 04-<09)$ & 01/01 & 100 & $(\geq 04-<09)$ & $01 / 01$ & 100 \\
\hline$(\geq 09-<14)$ & $04 / 04$ & 100 & $(\geq 09-<14)$ & $04 / 01$ & 25 \\
\hline$(\geq 14-\leq 18)$ & 03/03 & 100 & $(\geq 14-\leq 18)$ & $03 / 02$ & 66,7 \\
\hline Ferro (mg) & 03/10 & 30 & Vitamina C (mg) & $10 / 06$ & 60 \\
\hline Faixa etária & & & Faixa etária & & \\
\hline$(\geq 01-<04)$ & 00/02 & 00 & $(\geq 01-<04)$ & $02 / 00$ & 00 \\
\hline$(\geq 04-<09)$ & 01/01 & 100 & $(\geq 04-<09)$ & $01 / 00$ & 00 \\
\hline$(\geq 09-<14)$ & 00/04 & 00 & $(\geq 09-<14)$ & $04 / 03$ & 75 \\
\hline$(\geq 14-\leq 18)$ & $02 / 03$ & 66,7 & $(\geq 14-\leq 18)$ & $03 / 03$ & 100 \\
\hline Sódio (mg) & $06 / 10$ & 60 & Vitamina D (mcg) & $10 / 10$ & 100 \\
\hline Faixa etária & & & Faixa etária & & \\
\hline$(\geq 01-<04)$ & 00/02 & 00 & $(\geq 01-<04)$ & $02 / 02$ & 100 \\
\hline$(\geq 04-<09)$ & 01/01 & 100 & $(\geq 04-<09)$ & $01 / 01$ & 100 \\
\hline$(\geq 09-<14)$ & $02 / 04$ & 50 & $(\geq 09-<14)$ & $04 / 04$ & 100 \\
\hline$(\geq 14-\leq 18)$ & 03/03 & 100 & $(\geq 14-\leq 18)$ & $03 / 03$ & 100 \\
\hline Potássio (mg) & $10 / 10$ & 100 & Vitamina $\mathrm{E}(\mathrm{mg})$ & $10 / 10$ & 100 \\
\hline Faixa etária & & & Faixa etária & & \\
\hline$(\geq 01-<04)$ & $02 / 02$ & 100 & $(\geq 01-<04)$ & $02 / 01$ & 50 \\
\hline$(\geq 04-<09)$ & 01/01 & 100 & $(\geq 04-<09)$ & 01/01 & 100 \\
\hline$(\geq 09-<14)$ & $04 / 04$ & 100 & $(\geq 09-<14)$ & $04 / 03$ & 75 \\
\hline$(\geq 14-\leq 18)$ & 03/03 & 100 & $(\geq 14-\leq 18)$ & $03 / 03$ & 100 \\
\hline Zinco (mg) & $05 / 10$ & 50 & Ácido fólico (mcg) & $10 / 08$ & 80 \\
\hline Faixa etária & & & Faixa etária & & \\
\hline$(\geq 01-<04)$ & 00/02 & 00 & $(\geq 01-<04)$ & 02/01 & 50 \\
\hline$(\geq 04-<09)$ & 01/01 & 100 & $(\geq 04-<09)$ & $01 / 01$ & 100 \\
\hline$(\geq 09-<14)$ & $01 / 04$ & 25 & $(\geq 09-<14)$ & $04 / 04$ & 75 \\
\hline$(\geq 14-\leq 18)$ & 03/03 & 100 & $(\geq 14-\leq 18)$ & $03 / 03$ & 100 \\
\hline
\end{tabular}

* Número de indivíduos com inadequação para cada categoria.

** Número total de indivíduos por categoria.

\section{DISCUSSÃO}

A frequência de distribuição dos tipos de MPS dos pacientes avaliados nesse estudo foi semelhante à encontrada por Vieira et al. ${ }^{18}$ em 2008; de 113 indivíduos com MPS no Brasil, foi observada menor prevalência da MPS I (18 pacientes) e maior dos tipos II e VI (43 e 29 pacientes, respectivamente). Em um estudo realizado na cidade de Monte Santo (Bahia/ Brasil) a MPS tipo VI apresentou um número de casos muito maior do que descrito pela literatura, com mais de um caso para cada 5.000 habitantes. ${ }^{5}$ Segundo o 
mesmo estudo a MPS VI é considerada uma doença de grande impacto sobre a população de Monte Santo devido à endogamia elevada nesta região. O serviço em que foi realizado o estudo é referência em acompanhar pacientes com essa patologia em todo o estado da Bahia. $^{5}$

A Rede MPS Brasil, uma iniciativa criada para melhorar o diagnóstico e gestão destas doenças no Brasil, identificou, entre 2004 e 2006, 161 pacientes com MPS, entre os quais o tipo II mostrou maior prevalência $(n=82)$ e o tipo VII o menor número de casos $(n=4) .{ }^{19}$ Estudos sugerem que as MPSs tipo II e VI sejam as mais frequentemente diagnosticadas no país, sendo desconhecidas as explicações para tal achado, e observada maior frequência no Sudeste seguida da região Nordeste. ${ }^{1,2}$ No estudo de Villas-Boas et al., ${ }^{20}$ de 2011, realizado no mesmo centro de referência do presente estudo, de 29 pacientes avaliados, 11 (38\%) tinham diagnóstico de MPS tipo II, e o sexo masculino foi o mais prevalente, perfazendo $82,8 \%$ do grupo total. A alta prevalência do sexo masculino foi provavelmente influenciada pelo grande número de pacientes com MPS II, o qual tem herança recessiva ligada ao cromossomo $\mathrm{X} .{ }^{20}$

A idade dos pacientes avaliados foi similar à idade encontrada no estudo de Pinto et al. ${ }^{2}$ que descreveu uma média de idade dos pacientes de 7,3 anos (desvio padrão $=3,6$ ) na primeira consulta, e na segunda visita, de 8,4 anos (desvio padrão $=3,6) .^{2}$

Considerando as possíveis mudanças na consistência de alimentos e na autonomia no ato de se alimentar, na pesquisa de Turra e Schwartz ${ }^{21}$ a consistência dos alimentos ingeridos apresentou-se normal em 85,7\% (66/77) pacientes, semelhante a este estudo. ${ }^{21}$ Dos pacientes acompanhados neste estudo, a maior parte apresentou dificuldade em usar utensílios (garfo, faca, colher, copos), precisando de auxílio para se alimentar, o que pode interferir no consumo alimentar e consequentemente no estado nutricional desses pacientes. A baixa densidade energética pode estar relacionada ao tipo de alimento ingerido e à consistência do mesmo, tais como as preparações de consistência líquida e pastosa como sopas, mingaus e leite muito diluído. Quando é indicado ao paciente modificação da consistência alimentar, é essencial complementar o aporte de energia de acordo com as recomendações e necessidades do indivíduo, com uso de outros alimentos complementares ou suplementos nutricionais. ${ }^{22}$ Esta observação sinaliza a importância da educação nutricional, durante o acompanhamento de pacientes com MPS, direcionada para os responsáveis, com ênfase na necessidade de acompanhamento e auxílio dos pacientes no momento da alimentação, atentando para as possíveis dificuldades, como garantia da segurança alimentar e nutricional desses indivíduos.

Com relação à avaliação do consumo alimentar, recomenda-se uma investigação detalhada dos hábitos alimentares, incluindo o padrão alimentar quanto ao número, ao tipo e à composição das refeições, considerando a avaliação das condições alimentares da família quanto à renda e a disponibilidade de alimentos, bem como os aspectos culturais e socioeconômicos. ${ }^{23}$

De acordo com os dados do Censo 2010 do Instituto Brasileiro de Geografia e Estatística (IBGE), as regiões Norte e Nordeste apresentaram os maiores percentuais de pessoas que ganham até um salário mínimo como remuneração mensal de trabalho. ${ }^{24}$ No presente estudo observou-se que grande parte dos pacientes vivia com uma renda familiar menor ou igual a dois salários mínimos, o que pode impactar negativamente na aquisição de alimentos. Segundo Borges et al. ${ }^{25}$ a relevância do nível de renda na determinação de doenças parece decorrer do amplo efeito que esta exerce sobre a quantidade e a qualidade dos alimentos consumidos, corroborando a relação entre renda familiar e a ingestão alimentar. ${ }^{25}$ Neste trabalho observou-se que a maioria das genitoras tinha frequentado pelo menos o ensino fundamental, enquanto para os genitores houve uma frequência equilibrada entre analfabetismo, nível fundamental, médio e superior. Conforme o IBGE o percentual de pessoas sem instrução vem decaindo na última década. ${ }^{24}$

Considerando a frequência de inadequações na ingestão alimentar dos macronutrientes, a análise do estudo assemelha-se aos dados apresentados pela Pesquisa de Orçamentos Familiares (POF), realizada entre os anos de 2008 e 2009. ${ }^{26}$ A POF avaliou as médias de ingestão de energia, macronutrientes e micronutrientes nas grandes regiões do Brasil. $\mathrm{Na}$ região Nordeste, a participação calórica dos lipídios obteve valores abaixo dos valores nacionais, com a ingestão proteica ultrapassando o limite máximo recomendado. No presente estudo observamos que houve também um baixo consumo de lipídios, estando adequado o consumo de carboidratos e proteínas.

De acordo com a POF, o percentual da população com consumo de fibras abaixo do recomendado foi elevado (68\%), reflexo da alimentação baseada em cereais refinados e quantidades diminuídas de frutas, verduras e cereais integrais. No presente estudo todos os pacientes avaliados também apresentaram um consumo abaixo das recomendações desse nutriente. As prevalências de inadequação da ingestão de micronutrientes foram altas em todas as 
grandes regiões do país segundo os dados da POF, refletindo a baixa qualidade da dieta do brasileiro, com o baixo consumo de frutas, verduras, legumes e leite e derivados. As vitaminas A, E, C e D, e alguns minerais como cálcio, foram os nutrientes de maior percentual de inadequação, semelhantes aos resultados encontrados no presente estudo, com $60 \%$ dos pacientes apresentando ingestão abaixo da recomendação de cálcio, bem como uma ingestão inadequada de todas as vitaminas nas diferentes faixas etárias, excetuando-se as vitaminas do complexo B.

O suprimento nutricional abaixo das necessidades recomendadas tem consequências mais relevantes em organismos em desenvolvimento/crescimento, como nas crianças e adolescentes, devido à imaturidade nas vias metabólicas e das funções fisiológicas, o que poderá ser potencializado na presença de uma doença crônica. ${ }^{27}$ As alterações comuns da MPS, já descritas na literatura como nos lábios, língua, arcada dentária e mandíbula, bem como as alterações osteoarticulares, contribuem para um comprometimento importante de atos simples da vida prática, como vestirse e alimentar-se, contribuindo com possíveis mudanças na alimentação e no estado nutricional desses pacientes. ${ }^{28}$

A terapia nutricional associada à correção das alterações metabólicas e das deficiências de micronutrientes torna-se parte indispensável do tratamento dos pacientes com MPS, de forma a melhorar a evolução da doença e também a qualidade de vida dessa população. ${ }^{29} \mathrm{~A}$ inserção do trabalho do profissional de Nutrição na MPS amplia a visão curativa dos problemas sistêmicos de caráter crônico e progressivo, para uma perspectiva mais abrangente do indivíduo e da doença, visando avaliar as alterações fisiológicas, anatômicas e funcionais que alteram ou prejudicam a adequada nutrição desses pacientes. Cabe ao nutricionista elaborar e planejar de forma individualizada a assistência dietoterápica hospitalar ou ambulatorial necessária, prescrevendo, planejando, analisando, supervisionando as dietas, respeitando o alcance da oferta de todos os grupos de alimentos, com macronutrientes e micronutrientes adequados, volume e consistência da refeição ideal, com intuito de promover hábitos alimentares saudáveis, na prevenção de complicações, contribuindo com o tratamento dessa patologia, minimizando seu impacto sobre o estado nutricional. ${ }^{9}$

Diversos estudos já apontam para a influência positiva das estratégias adequadas de alimentação na infância, na saúde em longo prazo e na expectativa de vida da população. ${ }^{27}$ É clara a importância dos ajustes, monitoramento nutricional e do atendimento especializado no tratamento de diferentes doenças crônicas já descritas na literatura, no entanto na MPS, em virtude da escassez de estudos científicos, somente foi possível correlacionar os achados deste trabalho com os dados gerais da população brasileira, tornando relevante e evidente a necessidade de maiores investimentos em pesquisas científicas nesta área da saúde e com esta população, por períodos de tempo mais prolongados, a fim de gerarmos maiores conhecimentos sobre o impacto da nutrição na evolução da doença e na qualidade de vida do indivíduo com MPS.

Os resultados apresentados neste estudo apontam para uma elevada frequência de inadequações alimentares de pacientes com MPS, destacando-se a ingestão inadequada de calorias totais, lipídios, fibras e micronutrientes nas diferentes faixas etárias, resultados semelhantes aos encontrados na população brasileira com ausência de fatores limitantes. Por outro lado, o estudo evidenciou a existência de importantes fatores limitantes da capacidade funcional, que acabam contribuindo para afetar negativamente a nutrição dos pacientes com MPS.

Observou-se, na maioria dos casos, necessidade de auxílio durante as refeições e modificações dietéticas concernentes à consistência e ao volume. Intervenções nutricionais especializadas e melhora nas condições gerais de vida são imprescindíveis para o atendimento adequado das necessidades nutricionais desses pacientes, levando em conta as limitações individuais e visando a melhora na sua qualidade de vida e no seu prognóstico.

\section{REFERÊNCIAS}

1. Pereira JO, Garbelini MGAM, Palazzo VC. Mucopolysaccharidosis type VI: natural history, diagnostic and therapeutic importance. Rev Neurocienc. 2011;19(2):329-38.

2. Pinto LL, Schwartz IV, Puga AC, Vieira TA, Munoz MV, Giugliani R. Prospective study of 11 Brazilian patients with mucopolysaccharidosis II. J Pediatr (Rio J). 2006;82(4):273-8

3. Giugliani R, Federhen A, Rojas MVM, Vieira TAI, Artigalás O, Pinto LLC. Terapia de reposição enzimática para as Mucopolissacaridoses I, II e VI: Recomendações de um grupo de especialistas brasileiros. Rer Assoc Med Bras. 2010;56(3):257-77. 
4. Kakkis E, Wraith ED. Clinical features and diagnosis of the mucopolysaccharidoses. UpToDate Website 19.2 [updated 2011 Aug 10 ; cited 2011 Jun 15]. Available from: http://www.uptodate.com.

5. Costa-Motta FM, Acosta AX, Abé-Sandes K, Bender F, Schwartz IVD, Giugliani R, Leistner-Segal S. Genetic studies in a cluster of Mucopolysaccharidosis Type VI patients in Northeast Brazil. Mol Genet Metab. 2011;104:603-7.

6. Azevedo ACMM. Mucopolissacaridose tipo VI: um estudo clínico e radiológico visando a identificação de fatores associados à gravidade da doença [tese]. Porto Alegre (RS): UFRGS; 2007.

7. Informações ao Paciente: A Síndrome de Hunter, Mucopolissacaridose tipo II (MPS II). Shire Human Genetic Therapies [Internet]; 2007 [cited 2011 Nov 30]. Available from: http://www.hunterpatients.com.br.

8. Giugliani R, Harmatz P, Wraith JE. Management Guidelines for Mucopolysaccharidosis VI. Pediatrics. 2007;120(2):405-18.

9. Brasil. Conselho Federal de Nutricionistas. Resolução N 304. Dispõe sobre critérios para prescrição dietética na área de Nutrição Clínica e da outras providências. Brasília (DF): CFN; 2003.

10. Lohman TG, Roche AF, Martorell R. Anthropometric standardization reference manual. Champaign: Human Kinetics Books; 1988.

11. Vannucchi H, Monteiro JP, Chiarello P. Consumo alimentar: visualizando porções. Rio de Janeiro: Guanabara Koogan; 2007.

12. Fisberg RM, Slater B, Marchioni DML, Martini LA. Inquéritos alimentares: métodos e bases científicas. Barueri. São Paulo: Manole; 2005.

13. Avanutri \& Nutrição Serviços e Informática. Software de nutrição - Avanutri, versão 3.1; 2008.

14. Food and Nutrition Board. Institute of Medicine. National Academies. Dietary Reference Intakes (DRIs): recommended dietary allowances and adequate intakes [Internet]; 2005 [cited 2012 May 20]. Available from: http://www.nap.edu.

15. EpiData version 3.1. A comprehensive tool forvalidaded entry and documentation of data. Odense: The EpiData Association; $2003-2004$.

16. Ludwig DA. Use and misuse of p-values in designed and observational studies: guide for researchers and reviewers. Aviat Space Environ Med. 2005;76(7):675-80.

17. R Devopment Core Team. R: a language and environment for statistical computing [Internet]. Vienna: R Foundation for Statistical Computing; 2012. [cited 2012 May 20]. Available from: http://www.R-project.org.

18. Vieira T, Schwartz I, Muñoz V, Pinto L, Steiner C, Ribeiro M, Boy R, Ferraz V, de Paula A, Kim C, Acosta A, Giugliani R. Mucopolysaccharidoses in Brazil: what happens from birth to biochemical diagnosis? Am J Med Genet A. 2008;146A(13):1741-7.

19. Viana GM, de Lima NO, Cavaleiro R, Alves E, Souza IC, Feio R, Leistner-Segal S, Schwartz I, Giugliani R, da Silva LC. Mucopolysaccharidoses in northern Brazil: Targeted mutation screening and urinary glycosaminoglycan excretion in patients undergoing enzyme replacement therapy. Genet Mol Biol. 2011;34(3):410-5.

20. Villas-Bôas FS, Fernandes Filho DJ, Acosta AX. Ocular findings in patients with mucopolysaccharidosis. Arq Bras Oftalmol. 2011;74(6): 430-4.

21. Turra GS, Schwartz IV. Evaluation of orofacial motricity in patients with mucopolysaccharidosis: a cross-sectional study. J Pediatr (Rio J). 2009;85(3):254-60.

22. Brasil. Ministério da Saúde. Guia alimentar para crianças menores de dois anos. Brasília (DF): Ministério da Saúde; 2002.

23. Fisberg RM, Marchioni DM, Colucci AC. Assessment of food consumption and nutrient intake in clinical practice. Arq Bras Endocrinol Metabol. 2009;53(5):617-24

24. Instituto Brasileiro de Geografia e Estatística. Censo demográfico de 2010, resultados gerais da amostra [Internet] [cited 2012 May 20]. Available from: http://www.ibge.gov.br.

25. Borges CQ, Silva Rde C, Assis AM, Pinto Ede J, Fiaccone RL, Pinheiro SM. Factors associated with anemia in children and adolescents in public schools in Salvador, Bahia State, Brazil. Cad Saude Publica. 2009;25(4):877-88.

26. Instituto Brasileiro de Geografia e Estatística. Pesquisa de orçamentos familiares 2008-2009: análise do consumo alimentar pessoal no Brasil. Rio de Janeiro: IBGE; 2011.

27. Sabotka L. Bases da nutrição clínica. In: Kaletzo B. Necessidades nutricionais de crianças e adolescentes. $3^{\text {a }}$ ed. Rio de Janeiro: Rubio; 2008. p. 38-40.

28. Cardoso-Santos A, Azevedo AC, Fagondes S, Burin MG, Giugliani R, Schwartz IV. Mucopolysaccharidosis type VI (Maroteaux-Lamy syndrome): assessment of joint mobility and grip and pinch strength. J Pediatr (Rio J). 2008;84(2):130-5.

29. Mizuno CA, Figueiredo JB, Teza ITV, Taira LGN, Silva TA, Paixão DL, Mizuno JC. Clinical aspects of mucopolysaccharidosis type VI. Rev Bras Clin Med. 2010;8(4):356-61.

30. Sabotka L. Bases da nutrição clínica. In: Dardai E. Nutrição nas doenças cardíacas e pulmonares. $3^{\text {a }}$ ed. Rio de Janeiro: Rubio; 2008. p. 371-3. 